\title{
Soft-Output Maximum-Likelihood Detector for Quadrature Spatial Modulation
}

\author{
M. A. Tijani ${ }^{*}$, A. A. Tijani ${ }^{2}$ \\ ${ }^{1}$ Department of Electrical/Electronic Engineering Technology, Federal Polytechnic, Ede, Nigeria. \\ ${ }^{2}$ School of Electrical \& Information Engineering, University of the Witwatersrand, Johannesburg, South Africa.
}

\begin{abstract}
Spatial modulation (SM) presents a pragmatic approach for transmitting information, where the modulator uses well known amplitude/phase modulation (APM) techniques such as phase shift keying (PSK) and quadrature amplitude modulation (QAM), but also employs the antenna index to convey additional information. Nevertheless, the spectral efficiency of SM can be further improved. On this note, to further enhance the spectral efficiency of SM, quadrature SM (QSM) modulation was proposed. In coded channels, therefore, typically soft-output detection coupled with soft-input channel decoding yields significant signal-to-noise ratio (SNR) gain. Hence, in this paper, soft-output maximum-likelihood detector (SOMLD) for QSM modulated system is proposed. Monte Carlo simulation results demonstrate that there is a close agreement between the error performances of the proposed SOMLD scheme and those of its hard-decision maximum-likelihood detector counterpart in uncoded channels, while significant signal-to-noise ratio (SNR) gains are yielded in coded channels.
\end{abstract}

KEYWORDS: Multiple-input multiple-output, quadrature spatial modulation, soft-output detection, space shift keying, spatial modulation.

[Received February 05, 2018; Revised March 23, 2018; Accepted May 01, 2018]

Print ISSN: 0189-9546 | Online ISSN: 2437-2110

\section{INTRODUCTION}

Currently, much effort is being geared towards 5th generation $(5 \mathrm{G})$ wireless systems, in the research community. According to $\mathrm{Hu}$ and Qian (2014), the demands for 5G is motivated by increased capacity and data rates, improved quality of service, reduced latency and energy efficiency. Multiple-input multiple-output (MIMO) systems, as reported by Telatar (1999), hold the potential to meet these demands, but not without challenges. For example: the need for interantenna synchronization (IAS) between transmit antennas, increased form-factor allowing for ideal spacing of transmit and receive antennas, reduction of inter-channel interference (ICI) at the receiver and large computational complexity (CC) overhead. A number of schemes have recently been investigated to address these challenges, while exploiting the advantages of MIMO.

Mesleh et al. (2006) proposed spatial modulation (SM). The key idea in SM is to employ the index of a single transmit antenna as a means to convey additional information. Information is divided into two parts. The first part is mapped to a chosen symbol from an amplitude and/or phase modulation (APM) signal constellation, while the remaining part determines the transmit antenna that is to be activated for transmission of the APM symbol. It should be noted that the dormant antennas transmit zero power during each and every transmission. It was concluded by Jeganathan et al. (2008), Mesleh et al. (2008) and Naidoo et al. (2011); that SM completely avoids IAS, ICI at the receiver, and only requires a single radio frequency $(\mathrm{RF})$ chain, which translates into a relatively low-complexity receiver.

*Corresponding author's e-mail address: muhammedtijani@gmail.com
Based on these merits, the SM scheme proves to be a good candidate for deployment in next generation wireless communication systems. However, Jeganathan et al. (2009) opined that options for decreasing its system complexity exist; and on this note, a variant of SM in the form of space shift keying (SSK) was proposed. In SSK, only the spatial domain of SM is exploited to relay information. The elimination of the APM results in lowered detection complexity, less stringent transceiver requirements, and simplicity. A criticism of SSK is that large antenna arrays are required to achieve high data rates (Liang et al., 2012). In this regard, bi-space shift keying (BiSSK) was proposed by Liang et al. (2012) as an extension to SSK. Bi-SSK employs dual antenna indices (one associated with a real number and the other with an imaginary number) to carry information. This results in twice the achievable data rate of SSK, while preserving the advantages of the latter.

To improve the data rate of SM, quadrature SM (QSM) was proposed by Mesleh et al. (2014). In QSM, the overall throughput of SM is enhanced by extending its spatial domain into in-phase and quadrature-phase dimensions. In order to allow this, the complex constellation symbol of SM is further decomposed into its real and imaginary parts. The in-phase and quadrature-phase spatial dimensions are orthogonal cosine and sine carrier signals and are used for conveying the real and imaginary parts, respectively, of the APM transmission symbol. This, consequently, allows for an additional base-two logarithm of the number of transmit antennas bits to be transmitted; while other SM advantages, such as the usage of single RF chain at the transmitting end, avoidance of ICI, and low-complexity receiver, are preserved (Mesleh et al., 2014, Mesleh et al., 2015 and Naidu et al., 2015).

doi: http://dx.doi.org/10.4314/njtd.v15i4.5 
In practice, the majority of communication systems employ channel coding (Sklar, 2001). Moreover, it has been demonstrated in various and different researches conducted by Moon (2005), Hwang et al. (2009), Tang et al. (2013), Govender et al. (2014), Xiao et al. (2016), Zheng et al. (2017), $\mathrm{Li}$ et al. (2016) and Zhao et al. (2017); that a combination of soft-output detection with soft-input channel decoding results in a net coding gain compared to the conventional harddecision detection/decoding. For example, a soft-input softoutput detector, based on a block minimum mean-squared error algorithm, was proposed for generalised SM (GSM) by Xiao et al. (2016). In a similar research, two soft-input softoutput algorithms that are based on the deterministic sequential Monte Carlo (SMC) technique are proposed for demodulation of GSM signals (Zheng et al., 2017). These algorithms achieve near-optimal performances with low complexity. Moreover, turbo and recursive systematic convolutional (RSC) coded receiver are respectively employed to obtain better error performances for the GSM.

By exploiting the features of M-PSK and M-QAM constellations, an efficient transmission scheme for SM systems with soft-decision aided detector was presented by $\mathrm{Li}$ et al. (2016). The error correction ability of channel coding is exploited and low-density parity-check code is employed. Results show that the PSK and QAM-based soft-output detectors achieve the same performance as Max-Log-LLR algorithm with reduced CC. Furthermore, a study of the benefits of nonlinear frequency domain turbo equalizer (FDTE) in single-carrier-SM systems was carried out by Zhao et al. (2017). With a RSC coded receiver, the FDTE is aided by a time-domain soft-decision feedback (TDSDF) such that the equalizer operates on a frame-by-frame basis and is designed based on the minimum block-averaged mean-square error (MBMSE) criterion. Simulation results show that the proposed equalizer outperforms conventional linear FDTD.

In Moon (2005), Hwang et al. (2009), Tang et al. (2013) and Govender et al. (2014), respectively; soft-output detection algorithms for orthogonal frequency division multiplexing based SM (SM-OFDM) and space-time block coded SM were proposed, and significant signal-to-noise ratio (SNR) gains were demonstrated.

However, no such detectors have been proposed for the more recent variant of SM (i.e QSM). Hence, to address the demands of 5G, QSM also holds the potential for unleashing the benefits of MIMO. 5G systems will most certainly be adaptive (based on channel conditions). But certain modes will utilize channel coding. In these modes, the error performance may be boosted by employing soft-output detection coupled with soft-input channel decoding for the QSM scheme. In addition, the scheme could also be easily extended to LTEadvanced systems, such as in Li et al. (2012).

In this paper, soft-output detector for QSM modulated signals is proposed. This is motivated, as reported in Moon (2005), Hwang et al. (2009), Tang et al. (2013) and Govender et al. (2014), by the improved error rates that can be achieved from soft-output detection coupled with soft-input channel decoding. In summary, the contributions of the paper are as follows: a) A soft-output maximum-likelihood detector
(SOMLD) for QSM is formulated, b) a detailed analysis of the $\mathrm{CC}$ for the proposed detector is presented and c) Monte Carlo simulations for the proposed detector are demonstrated. The remainder of the paper is organized such that Section 2 presents the QSM system model while the proposed softoutput detector is presented in Section 3. Analysis of the CC of the new detection is presented in Section 4, discussion of simulation results is given in Section 5, and finally, conclusions are drawn in Section 6.

Notation: Matrices are denoted with bold italics uppercase letters, vectors with bold italics lowercase letters and scalars with regular letters. $(\cdot)^{\mathrm{T}}$ is used for transpose, $(\cdot)^{\mathrm{H}}$ for Hermitian and $\|\cdot\|_{F}$ for the Frobenius norm of a vector or matrix. $\mathfrak{K}\{\cdot\}$ and $\mathfrak{I}\{\cdot\}$ are used to represent the real and imaginary parts of a complex number, $j=\sqrt{-1}$ is a complex number and $\underset{x}{\operatorname{argmin}} f(x)$ for argument of the minimum, which returns a set of values of $x$ for which the function $f(x)$ attains its smallest value.

\section{QSM SYSTEM MODEL}

As presented by Mesleh et al. (2014), the rule governing the transmission of QSM stipulates that a group of $m_{Q S M}=$ $\log _{2}\left(N_{t}^{2} M\right)$ information bits can be transmitted simultaneously. A model of the QSM system is depicted in Figure 1. The source information bit sequence is partitioned into three parts, such that $\log _{2}\left(N_{t}\right)$ bits are used to select the real antenna index $\left(\ell_{\mathfrak{K}}\right)$, another $\log _{2}\left(N_{t}\right)$ bits are used to select the imaginary antenna index $\left(\ell_{\mathfrak{T}}\right)$ for $\ell_{\mathfrak{K}}, \ell_{\mathfrak{T}} \in\left[1: N_{t}\right]$. The remaining $\log _{2}(M)$ bits are used to select an $M$-ary quadrature amplitude modulation (MQAM) constellation symbol $x^{q}, q \in$ [1: $M]$.

The selected symbol $x^{q}$ of the complex constellation is further decomposed into its constituent real and imaginary parts $x^{q \mathfrak{K}}, x^{q \mathfrak{I}}$, respectively, and transmitted by the respective antennas. Thus, the signal vector of QSM can be represented as:

$$
x_{\mathrm{QSM}}=x_{\ell_{\mathfrak{K}}}^{q \mathfrak{K}}+j x_{\ell_{\mathfrak{I}}}^{q \mathfrak{I}}
$$

where $\boldsymbol{x}_{\ell_{\mathfrak{K}}}^{q \mathfrak{K}}$ and $\boldsymbol{x}_{\ell_{\mathfrak{T}}}^{q \mathfrak{I}}$ are $N_{t} \times 1$ vectors, with $x^{q \mathfrak{K}}$ and $x^{q \mathfrak{I}}$, respectively, representing the non-zero entry placed at the $\ell_{\mathfrak{K}^{-}}$ th and $\ell_{\mathfrak{T}}$-th positions.

An example of the mapping process for QSM modulation, is tabulated in Table 1, considering a $4 \mathrm{~b} / \mathrm{s} / \mathrm{Hz}$ QSM transmission with $N_{t}=2$ and $M=4$. In the example, the total number of transmitted information bits per time slot can be calculated as $\mathrm{m}_{\mathrm{QSM}}=\log _{2}\left(\mathrm{~N}_{\mathrm{t}}^{2} \mathrm{M}\right)=\log _{2}\left(2^{2} .4\right)=4$ bits. In accordance with the mapping rules of QSM; the set of 4 bits $\left(b_{3} b_{2} b_{1} b_{0}\right)$ are grouped into three [i.e. $\log _{2}\left(N_{t}\right)+\log _{2}\left(N_{t}\right)+$ $\left.\log _{2}(\mathrm{M})\right]$ such that the first and the second groups are used to active one antenna each while the third selects an APM symbol, respectively. The selected APM symbol is further decomposed to its real and imaginary components such that they are transmitted over the real and imaginary active antennas, accordingly. 


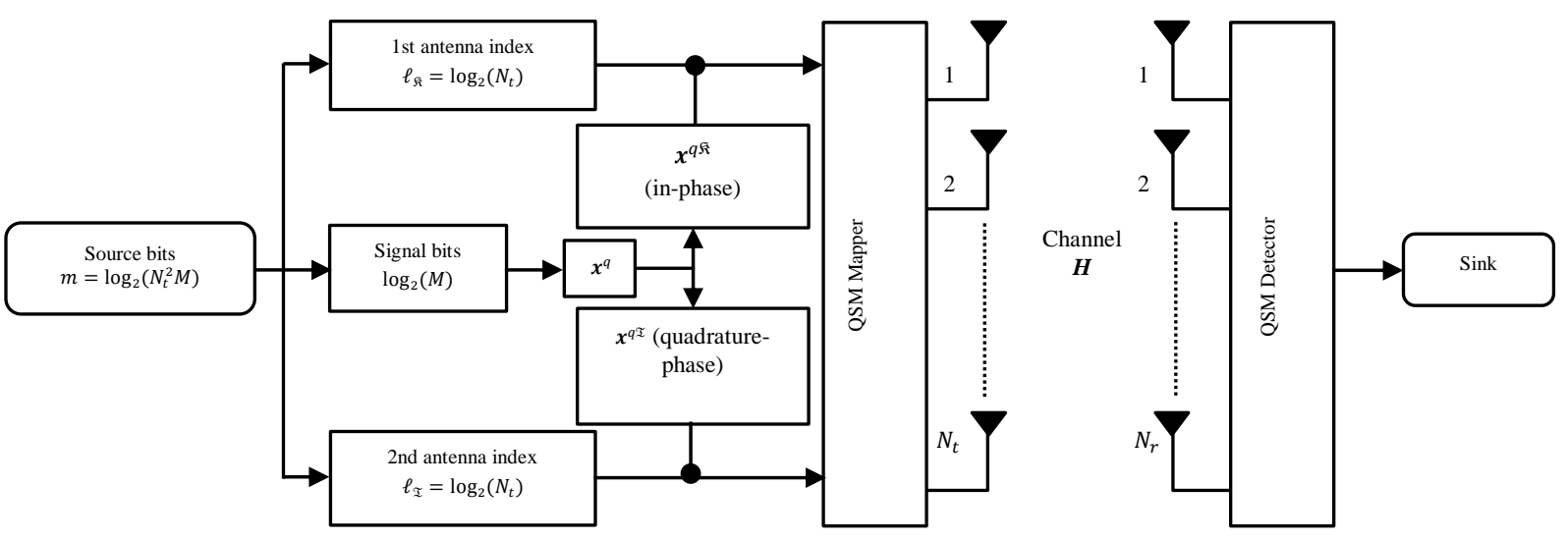

Figure 1: System Model for QSM Modulation.

Note, from the table, that some 12 other possible values of "Bits" have been omitted. Generally, this is done intentionally to save spaces, and because the omitted possibilities can easily be obtained from the given table. For example, when Bits = $\left[\begin{array}{llll}0 & 0 & 0 & 1\end{array}\right]$; the " $q$ " is " $00(1)$ " and this can be inferred from rows 3 of Table 1 , where the same bits, " 00 ", have been considered for " $q$ ". In the same way the " $\ell_{\mathfrak{K}}$ " and " $\ell_{\mathfrak{T}}$ " are " 0 " and " 1 ", respectively. Their corresponding antennas can also be inferred from row 2 of the table such that the active transmit antenna pair are " 1 " and " 2 ".

At the output of the channel, the received signal vector may be defined as:

$$
\boldsymbol{y}=\sqrt{\rho / \mu} \boldsymbol{H}\left(\boldsymbol{x}_{\ell_{\mathfrak{K}}}^{q \mathfrak{K}}+j \boldsymbol{x}_{\ell_{\mathfrak{T}}}^{q \mathfrak{T}}\right)+\boldsymbol{w}
$$

where $\mu$ is the scaling factor for the average SNR, such that when $\ell_{\mathfrak{K}}=\ell_{\mathfrak{T}}, \mu=1$, and when $\ell_{\mathfrak{K}} \neq \ell_{\mathfrak{T}}, \mu=2 . \boldsymbol{H}$ is the wireless channel with a complex channel matrix of dimension $N_{r} \times N_{t}$ in the presence of AWGN represented as $\boldsymbol{w}=$ $\left[w_{1}, w_{2}, \ldots, w_{N_{r}}\right]^{\mathrm{T}}$. The entries of $\boldsymbol{H}$ and $\boldsymbol{w}$ are assumed to be independent and identically distributed (i.i.d) according to $C N(0,1)$. The received signal may be simplified as:

$$
\boldsymbol{y}=\sqrt{\rho / \mu}\left(\boldsymbol{h}_{\ell_{\mathfrak{K}}} x^{q \mathfrak{r}}+j \boldsymbol{h}_{\ell_{\mathfrak{I}}} x^{q \mathfrak{I}}\right)+\boldsymbol{w}
$$

where $\boldsymbol{h}_{\ell_{\mathfrak{K}}}=\left[h_{1, \ell_{\mathfrak{K}}}, \ldots, h_{N r, \ell_{\mathfrak{K}}}\right]^{\mathrm{T}}$ represents the $N_{r} \times 1 \ell_{\mathfrak{K}}^{\text {th }}$ column of $\boldsymbol{H}$ and $\boldsymbol{h}_{\ell_{\mathfrak{T}}}=\left[h_{1, \ell_{\mathfrak{T}}}, \ldots, h_{N r, \ell_{\mathfrak{T}}}\right]^{\mathrm{T}}$ represents the $N_{r} \times 1 \ell_{\mathfrak{T}}^{\text {th }}$ column of $\boldsymbol{H}$.
Assuming perfect knowledge of the channel at the receiver, the received signals are processed by the optimum ML detector, which searches jointly across all the available antenna combinations and APM symbols. The detector jointly estimates $\hat{\ell}_{\mathfrak{K}}, \hat{\ell}_{\mathfrak{I}}, x^{\hat{q} \mathfrak{K}}$, and $x^{\hat{q} \mathfrak{I}}$, which are used to recover the original message. The detector may be given as:

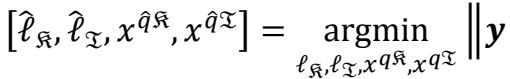

$$
\begin{aligned}
& -\sqrt{\rho / \mu}\left(\boldsymbol{h}_{\ell_{\mathfrak{K}}} x^{q \mathfrak{K}}+j \boldsymbol{h}_{\ell_{\mathfrak{I}}} x^{q \mathfrak{T}}\right) \|_{\mathrm{F}}^{2} \\
& \operatorname{LLR}\left(\ell_{\mathfrak{\kappa}}^{a}\right)=\log \left\{\frac{P\left(\ell_{\mathfrak{\kappa}}^{a}=1 \mid \boldsymbol{y}\right)}{P\left(\ell_{\mathfrak{K}}^{a}=0 \mid \boldsymbol{y}\right)}\right\}
\end{aligned}
$$

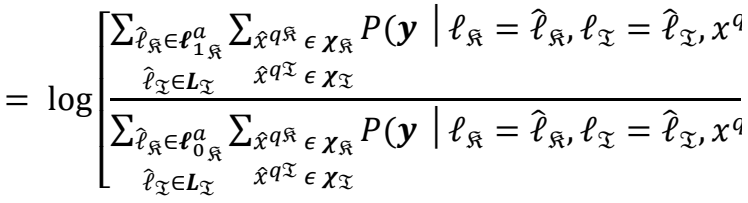

\begin{tabular}{|c|c|c|c|c|c|c|}
\hline \multirow[t]{2}{*}{$\begin{array}{c}\text { Bits } \\
\left(b_{3} b_{2} b_{1} b_{0}\right)\end{array}$} & \multicolumn{2}{|c|}{ APM symbol $\left(b_{3} b_{2}\right)$} & \multicolumn{2}{|c|}{$\begin{array}{c}\text { Tx. antenna pairs } \\
\left(\mathrm{b}_{1} / \mathrm{b}_{0}\right)\end{array}$} & \multirow{2}{*}{\multicolumn{2}{|c|}{$\begin{array}{c}x_{\mathrm{QSM}} \\
\left(x_{\boldsymbol{\ell}_{\mathfrak{K}}}^{q \mathfrak{K}}+j x_{\boldsymbol{\ell}_{\mathfrak{T}}}^{q \mathfrak{T}}\right)\end{array}$}} \\
\hline & $q($ index $)$ & $x^{q \mathfrak{K}}+j x^{q \mathfrak{I}}$ & $\ell_{\xi}$ & $\ell_{\mathfrak{T}}$ & & \\
\hline$\left[\begin{array}{llll}1 & 1 & 0 & 0\end{array}\right]$ & $11(4)$ & $+1-j$ & 1 & 1 & {$[+1-j]$} & $0]^{\mathrm{T}}$ \\
\hline$\left[\begin{array}{llll}1 & 0 & 0 & 1\end{array}\right]$ & $10(3)$ & $+1+j$ & 1 & 2 & {$[+1$} & $+j]^{\mathrm{T}}$ \\
\hline$\left[\begin{array}{llll}0 & 0 & 1 & 0\end{array}\right]$ & $00(1)$ & $-1+j$ & 2 & 1 & {$[+j$} & $-1]^{\mathrm{T}}$ \\
\hline$\left[\begin{array}{llll}0 & 1 & 1 & 1\end{array}\right]$ & $01(2)$ & $-1-j$ & 2 & 2 & {$[0$} & $-1-j]^{\mathrm{T}}$ \\
\hline
\end{tabular}

Table 1: Mapping illustration for QSM modulation. 


\section{PROPOSED SOFT-OUTPUT DETECTOR}

It is desired that next generation communication systems provide users with high data rates, in addition to ensuring reliability and power efficiency. To achieve this, practical communication systems commonly employ channel coding such that errors induced by noise and unreliable channels are reduced. It has been demonstrated by Tang (1991), Sklar (2001), Moon (2005) and Li et al. (2012), that soft-output detection coupled with soft-input channel decoding maximizes the coding gain achievable. In Tang et al. (2013) and Govender et al. (2014); soft-output detection has been investigated for SM and space-time block coded SM, respectively. However, no such investigation has been performed for the more recent QSM scheme, which maintain several advantages over SM.

Based on this motivation, SOMLD for the QSM scheme is proposed. To arrive at the targeted improvements in the error performances of the respective systems due to coding gain, the system model of Fig. 1 is extended to include channel coding and decoding. For the proposed detection scheme, we assume that:

i. antenna indices and data symbols are uncorrelated;

ii. data symbols are independent and generated with equal probability;

iii. antenna bits are independent and generated with equal probability, and;

iv. full channel knowledge is available at the receiver.

The codewords from the channel encoder are transmitted by QSM modulation, such that the input to our proposed demodulator is given as (2). The proposed SOMLD demodulator independently calculates the LLR for the $a$-th real antenna bit $\left(\ell_{\mathfrak{K}}^{a}\right), a$-th imaginary antenna bit $\left(\ell_{\mathfrak{I}}^{a}\right), b$-th real symbol bit $\left(x_{\mathfrak{K}}^{b}\right)$ and $b$-th imaginary symbol bit $\left(x_{\mathfrak{T}}^{b}\right)$. The aposteriori log-likelihood ratios (LLRs) in accordance with the work of Tang et al. (2013) may be formulated as follows:

Considering demodulation of the $a$-th real transmit antenna bit:

where $\boldsymbol{\ell}_{0 \mathfrak{K}}^{a}$ and $\boldsymbol{\ell}_{1 \mathfrak{K}}^{a}$ are vectors of the real antenna indices with ' 0 ' and ' 1 ', respectively, for the $a$-th antenna bit position, and $\chi_{\mathfrak{K}}, \chi_{\mathfrak{I}}$ represents the set of all possible $\hat{x}^{q \mathfrak{K}}$ and $\hat{x}^{q \mathfrak{I}}$, respectively.

On application of the Bayes' theorem, the demodulator output in (6) can be formulated as:

$$
\begin{aligned}
& \operatorname{LLR}\left(\ell_{\mathfrak{K}}^{a}\right)
\end{aligned}
$$

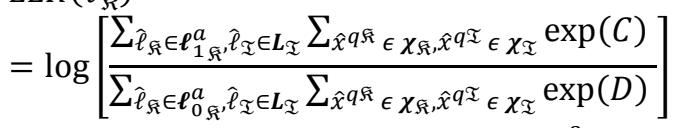

where $C=D=\frac{-\left\|y-\sqrt{\rho / \mu}\left(\boldsymbol{h}_{\hat{\ell}_{\mathfrak{K}}} \hat{x}^{q \mathfrak{\Re}}+j \boldsymbol{h}_{\hat{\ell}_{\mathfrak{T}}} \hat{x}^{q \mathfrak{T}}\right)\right\|_{\mathrm{F}}^{2}}{2 \sigma^{2}}$, with $\sigma^{2}$ the variance of the AWGN.

Similarly, the $a$-th imaginary antenna bit is computed and expressed as:

$$
\begin{aligned}
& \operatorname{LLR}\left(\ell_{\mathfrak{T}}^{a}\right)
\end{aligned}
$$

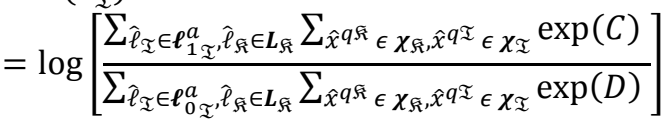

where $\boldsymbol{\ell}_{0 \mathfrak{I}}^{a}$ and $\boldsymbol{\ell}_{1 \mathfrak{T}}^{a}$ are vectors of the imaginary antenna indices with ' 0 ' and ' 1 ', respectively, at the $a$-th antenna bit position.

Furthermore, the $b$-th real symbol bit is computed as:

$$
\begin{aligned}
& \operatorname{LLR}\left(x_{b}^{q \mathfrak{K}}\right)=\log \left\{\frac{P\left(x_{b}^{q \mathfrak{K}}=1 \mid \boldsymbol{y}\right)}{P\left(x_{b}^{q \mathfrak{K}}=0 \mid \boldsymbol{y}\right)}\right\}
\end{aligned}
$$

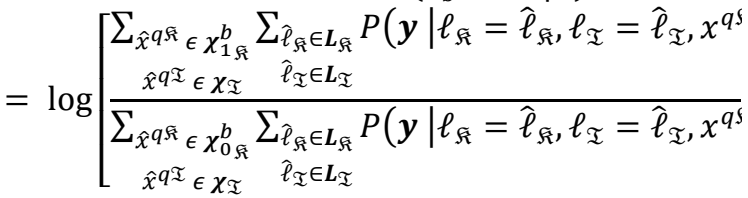

$$
\begin{aligned}
& \operatorname{LLR}\left(x_{b}^{q \mathfrak{K}}\right)
\end{aligned}
$$

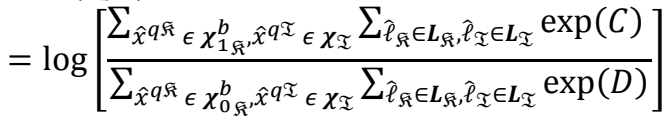

where $\chi_{0_{\mathfrak{K}}}^{b}$ and $\chi_{1_{\mathfrak{K}}}^{b}$ are vectors of the real data symbols with ' 0 ' and ' 1 ', respectively, at the $b$-th data bit position.

Similarly, the $b$-th imaginary symbol bit is computed as:

$$
\begin{aligned}
& \operatorname{LLR}\left(x_{b}^{q \mathfrak{I}}\right) \\
& =\log \left[\frac{\sum_{\hat{x}}^{q \mathfrak{I}} \epsilon \chi_{1_{\mathfrak{I}}, \hat{x}^{q \mathfrak{N}} \in \chi_{\mathfrak{K}}} \sum_{\hat{\ell}_{\mathfrak{K}} \in \boldsymbol{L}_{\mathfrak{K}}, \hat{\ell}_{\mathfrak{I}} \in \boldsymbol{L}_{\mathfrak{I}}} \exp (C)}{\sum_{\hat{x}}{ }_{\mathfrak{T}} \in \chi_{0 \mathfrak{I}}^{b}, \hat{x}^{q \mathfrak{N}} \in \chi_{\mathfrak{K}} \sum_{\hat{\ell}_{\mathfrak{K}} \in \boldsymbol{L}_{\mathfrak{K}}, \hat{\ell}_{\mathfrak{I}} \in \boldsymbol{L}_{\mathfrak{I}}} \exp (D)}\right]
\end{aligned}
$$

where $\chi_{0 \mathfrak{T}}^{b}$ and $\chi_{1_{\mathfrak{I}}}^{b}$ are vectors of the imaginary data symbols with ' 0 ' and ' 1 ', respectively, at the $b$-th data bit position.

The proposed soft-output detector achieve an improvement in error performance when the output is fed into a soft-input Viterbi channel decoder as described and used by Hwang et al. (2009), Tang et al. (2013) and Govender et al. (2014) and estimates of the transmitted messages are obtained.

\section{COMPARISON OF COMPUTATIONAL COMPLEXITIES}

In this section, the computational complexity $(C C \mathrm{~s})$ for the proposed SOMLD for QSM system is evaluated. For fair comparison, the complexity of its HDMLD counterpart is also mention. Particularly, in the analyses of the imposed $C C \mathrm{~s}$ of the proposed SOLMDs, the following assumptions are made:

i. only complex additions and multiplications are considered to have impacts on the complexity of the detectors. This approach is consistent with the approaches in Naidoo et al. (2011) and Govender et al. (2014). 
ii. The complexity imposed by all metrics are considered, including those that only need to be solved once and stored for future computations. Thus, precomputed results are available such that redundant computation is not required. This approach is consistent with Jeganathan et al. (2008), Naidoo et al. (2011) and Govender et al. (2014).

iii. Finally, the computation of the logarithm functions, present in the LLRs of the detectors, are approximated via the use of a look-up table (LUT)-based method presented by Tang (1991) and therefore impose no additional complexity.

To formulate the $C C$ of the QSM-SOMLD, the individual complexity imposed by each of the four detection processes of detecting the $a$-th real antenna bit, $a$-th imaginary antenna bit, $b$-th real symbol bit and $b$-th imaginary symbol bit, is analysed. With the expansion of the Frobenius norms in (7), (8), (11) and (12), $C=D=\left(\sqrt{\rho / \mu}\left\|\boldsymbol{h}_{\ell_{\mathfrak{\xi}}}\right\|_{F}^{2}\left|x_{\ell_{\mathfrak{\kappa}}}^{q \mathfrak{\xi}}\right|^{2}-\right.$ $2 \mathfrak{K}\left\{\boldsymbol{y}^{H} \boldsymbol{h}_{\ell_{\mathfrak{K}}} \boldsymbol{x}_{\ell_{\mathfrak{K}}}^{q \mathfrak{K}}\right\}+\sqrt{\rho / \mu}\left\|\boldsymbol{h}_{\ell_{\mathfrak{T}}}\right\|_{F}^{2}\left|\boldsymbol{x}_{\ell_{\mathfrak{I}}}^{q \mathfrak{T}}\right|^{2}-$ $\left.2 \mathfrak{K}\left\{\boldsymbol{y}^{H} \boldsymbol{h}_{\ell_{\mathfrak{I}}} \boldsymbol{x}_{\ell_{\mathfrak{I}}}^{q \mathfrak{I}}\right\}\right) / 2 \sigma^{2}$. Here we first consider the CC of $[C / D]$ as the common factor to all the detection processes to the QSM-SOMLD.

Considering the first term of the $C$ (as the numerator), $\left\|\boldsymbol{h}_{\ell_{\mathfrak{\kappa}}}\right\|_{F}^{2}$ is equivalent to $\boldsymbol{h}_{\ell_{\mathfrak{\kappa}}}{ }^{H} \boldsymbol{h}_{\ell_{\tilde{\kappa}}}$ and requires $2 N_{r}$ complex multiplications for each $\hat{\ell}_{\mathfrak{\Re}}$. Thus, computing $\left\|\boldsymbol{h}_{\ell_{\mathfrak{R}}}\right\|_{F}^{2}$ for $\hat{\ell}_{\mathfrak{K}} \in$ $\ell_{1_{\mathfrak{R}}}^{a}$ requires $2 N_{r}\left(\frac{c}{2}\right)$ complex multiplications, while the computation of $\left|x_{\ell_{\mathfrak{K}}}^{q \mathfrak{\xi}}\right|^{2}$ requires only $M$ complex multiplications. Therefore, the $C C$ imposed by the first term is given by $2 N_{r}\left(\frac{c}{2}\right)+M$. A consideration of the second term shows that the computation of $\left\|\boldsymbol{h}_{\ell_{\mathfrak{I}}}\right\|_{F}^{2}=\left\|\boldsymbol{h}_{\ell_{\tilde{\xi}}}\right\|_{F}^{2}\left\|\boldsymbol{h}_{\ell_{\S}}\right\|_{F}^{2}$, therefore, the computation of $\left\|\boldsymbol{h}_{\ell_{\mathfrak{I}}}\right\|_{F}^{2}$ is ignored and the stored result is used. This means that the computation of the second term $\sqrt{\rho / \mu}\left\|\boldsymbol{h}_{\ell_{\mathfrak{I}}}\right\|_{F}^{2}\left|\boldsymbol{x}_{\ell_{\mathfrak{I}}}^{q \mathfrak{I}}\right|^{2}$ is dependent only on the computation of $\left|\boldsymbol{x}_{\ell_{\mathfrak{I}}}^{q \mathfrak{I}}\right|^{2}$ which is given by $M$. The third term requires $2 N_{r}$ complex multiplications for $\boldsymbol{y}^{H} \boldsymbol{h}_{\ell_{\tilde{\kappa}}}$ and a further $M$ complex multiplications for $\boldsymbol{x}_{\ell_{\mathfrak{\kappa}}}^{q \mathfrak{K}}$, for each of the $\frac{c}{2}$ antennapair combinations; making a total of $\left(2 N_{r}+M\right)\left(\frac{c}{2}\right)$. Obviously, the computation of the forth term will be equivalent to $\left(2 N_{r}+M\right)\left(\frac{c}{2}\right)$. Hence, the CC imposed by the numerator is the sum of: $\left(2 N_{r}\left(\frac{c}{2}\right)+M\right)+M+\left(2 N_{r}+M\right)\left(\frac{c}{2}\right)+\left(2 N_{r}+\right.$ $M)\left(\frac{c}{2}\right)$. This is equivalent to: $\delta_{\text {numerator }}=(c)\left(3 N_{r}+M\right)+$ $2 M$.

Next, we consider $D$ (as the denominator). It is evident that the computation of the first term depends only on $\left\|\boldsymbol{h}_{\ell_{\mathfrak{\kappa}}}\right\|_{F}^{2}$ for $\hat{\ell}_{\mathfrak{K}} \in \ell_{0_{\mathfrak{K}}}^{a}$ which requires $2 N_{r}\left(\frac{c}{2}\right)$ complex multiplications. This is in line with the assumption that results can be stored and reused in future computations so as to avoid redundant computations. For the same reason, the second term does not impose any further complexity. The third term imposes $\left(2 N_{r}+M\right)\left(\frac{c}{2}\right)$ complex multiplications, while the forth term similarly requires $\left(2 N_{r}+M\right)\left(\frac{c}{2}\right)$ complex multiplications. Therefore, the $C C$ of the denominator is calculated as $\delta_{\text {denominator }}=(c)\left(3 N_{r}+M\right)$.

Following the assumption (iii) and in accordance with the analyses above, the $C C$ imposed by estimating the $a$-th real antenna bit $\left(\ell_{\mathfrak{s}}^{a}\right)$ in (22) is given by the addition of $\delta_{\text {numerator }}$ and $\delta_{\text {denominator }}$; which is $\delta_{\ell_{\Re}^{a}}=c\left(6 N_{r}+2 M\right)+2 M$. Using a similar approach, it can be shown that the $C C$ imposed by the estimation of the $a$-th imaginary antenna bit $\left(\ell_{\mathfrak{I}}^{a}\right), b$-th real symbol bit $\left(x_{\mathfrak{K}}^{b}\right)$, and $b$-th imaginary symbol bit $\left(x_{\mathfrak{I}}^{b}\right)$ will not impose any further complexities as stored results can reused in order to avoid redundant computations. Hence, the $C C$ of our proposed detector, in terms of complex multiplications, can be written as:

$$
C C_{\mathrm{QSM}-\mathrm{SOMLD}}=c\left(6 N_{r}+2 M\right)+2 M
$$

Meanwhile, in terms of complex multiplications involved in the detection process, the complexity imposed by jointly detecting $\hat{\ell}_{\mathfrak{K}}, \hat{\ell}_{\mathfrak{I}}, \widehat{\boldsymbol{x}}^{q \mathfrak{K}}$ and $\widehat{\boldsymbol{x}}^{q \mathfrak{I}}$ in (3) can be analysed, on assumptions in (i) and (ii). Let, (3) be represented as $\widehat{\boldsymbol{x}}=$ $\operatorname{argmin}[K]$ such that. $\ell_{\mathfrak{K},}, \ell_{\mathfrak{T}}, x^{q \mathfrak{K},}, x^{q \mathfrak{T}}$

$$
\begin{aligned}
K=\sqrt{\rho / \mu} \| \boldsymbol{h}_{\ell_{\mathfrak{K}}} & \left\|_{F}^{2}\left|x_{\ell_{\mathfrak{K}}}^{q \mathfrak{F}}\right|^{2}+\sqrt{\rho / \mu}\right\| \boldsymbol{h}_{\ell_{\mathfrak{I}}} \|_{F}^{2}\left|x_{\ell_{\mathfrak{I}}}^{q \mathfrak{T}}\right|^{2} \\
& -2 \mathfrak{K}\left\{\boldsymbol{y}^{\mathrm{H}} \boldsymbol{h}_{\ell_{\mathfrak{K}}} x_{\ell_{\mathfrak{K}}}^{q \mathfrak{K}}\right\} \\
& -2 \mathfrak{K}\left\{\boldsymbol{y}^{\mathrm{H}} \boldsymbol{h}_{\ell_{\mathfrak{I}}} x_{\ell_{\mathfrak{I}}}^{q \mathfrak{T}}\right\}
\end{aligned}
$$

Thus, the $C C$ of the QSM-HDMLD detector can be evaluated based on $K$ as the total complexities imposed by the joint detection of $\hat{\ell}_{\mathfrak{R}}, \hat{\ell}_{\mathfrak{I}}, \hat{x}^{q \mathfrak{r}}$ and $\hat{x}^{q \mathfrak{I}}$, in terms of complex multiplications. Here, the overall $C C$ is estimated and given as $C C_{\mathrm{QSM}-\mathrm{HDMLD}}=c\left(6 N_{r}+2 M\right)+2 M$ which is similar to $8 N_{r} 2^{m}$ given by Mesleh et al. (2014).

In the above, the $C C$ s for the proposed QSM-SOMLD and that of the conventional HDMLD receivers, are presented. A critical comparison of these $C C$ s shows that HDMLD and the proposed SOMLD have the same $C C$ s in each case. It can therefore be concluded that the proposed SOMLD, despite its impressive performance, impose no additional computational complexity compared to its conventional HDMLD counterpart. Remarkably, when compared with traditional polynomial/rational-function algorithms discussed in Cody and Waite (1980) and Hart (1968), the LUT - which has been employed in the $C C$ analyses of the proposed SOMLD, is faster - because it requires less work in the approximation steps, more accurate - because the rounding error made in the approximation is usually tiny, and amendable to tight error analysis. However, it is noted that logarithm functions are usually computed by using a Taylor series or another iterative algorithm - which takes up valuable clock cycles; hence, for 
the LUT method, the space complexity can be maintained at the cost of only a minimal increase in hardware.

\section{NUMERICAL RESULTS AND DISCUSSION}

Monte Carlo simulations of the proposed detectors were executed in the Matlab environment and are in terms of the average bit error rate (BER) as a function of the average SNR. The termination criterion for simulations was the number of bit errors, set at 3000. Simulations were run until a BER of $10^{-6}$. For all simulations, Rayleigh frequency-flat fading channels and the presence of AWGN is assumed. It is assumed that 4 antennas are employed at the receiver. For all coded cases, a $1 / 2$ rate convolutional encoder was employed to encode the information bits under the constraint length 9 with code generator matrices $g_{1}=(561)_{\text {octal }} ; g_{2}=(753)_{\text {octal }}$. At the receiver, the proposed detector is employed and its output is fed into a soft-input Viterbi channel decoder (Sklar, 2005 and Moon, 2005), in order to obtain estimates of the transmitted messages.

In the following investigations, two spectral efficiencies are considered, viz. $6 \mathrm{~b} / \mathrm{s} / \mathrm{Hz}$ and $4 \mathrm{~b} / \mathrm{s} / \mathrm{Hz}$. For each of these spectral efficiencies, a configuration, such as to satisfy $m_{Q S M}=\log _{2}\left(N_{t}^{2} M\right)$, is chosen. It should be noted that, if no channel coding is employed, then the SOMLD performance is expected to match that of the HDMLD. This is because both detectors are based on the ML principle and there is no additional coding gain that may be exploited, hence reducing to the same solution. This is in accordance with the research carried out by Sklar (2001) and Moon (2005). Thus, this is also considered as a means of validation.

In Figure 2, the error performances of HDMLD and SOMLD detectors are evaluated, both for coded and uncoded channel conditions for $6 \mathrm{~b} / \mathrm{s} / \mathrm{Hz}$ SSK systems. For uncoded channels, simulation results demonstrate that the proposed SOMLD scheme matches identically with the HDMLD. Hence, the soft-output demodulator has no effect, unless employed in a coded channel and coupled with a soft-input decoder at the receiver.

In the same figure, it is evident that the coded SOMLD detector yields significant SNR gains. For example, at a BER of $10^{-6}$, an SNR gain of approximately $4.7 \mathrm{~dB}$ is achieved over coded HDMLD. Hence, the advantage of soft-output demodulation followed by soft-input decoding is demonstrated. Moreover, for illustrative purposes, by the use of coding, the proposed SOMLD achieves an SNR gain of approximately $8.5 \mathrm{~dB}$ over the uncoded HDMLD/SOMLD scheme, at the same BER. This large gain is expected, since we are comparing a coded and uncoded system in this instance.

In Figure 3; the error performances of $4 \mathrm{~b} / \mathrm{s} / \mathrm{Hz}$ QSM $(2 \times 4)$ system is presented. The results are demonstrated under the proposed soft-output detector as compared to the existing HDMLD in coded and uncoded channels. Similar behaviour as shown for $6 \mathrm{~b} / \mathrm{s} / \mathrm{Hz}$ is evident, in all cases. The uncoded HDMLD and SOMLD curves are identical. When coding is employed, HDMLD yields an SNR gain of $3.9 \mathrm{~dB}$. The proposed SOMLD further enhance the SNR gain by $2.5 \mathrm{~dB}$.

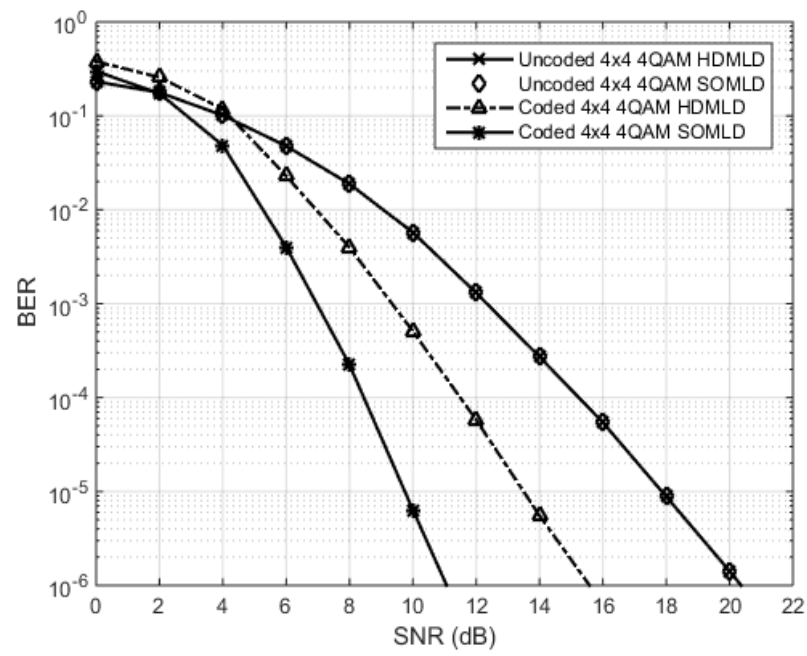

Figure 2: Comparison of error performances for $6 \mathrm{~b} / \mathrm{s} / \mathrm{Hz}$ QSM systems in coded and uncoded channels.

The smaller gain realized is due to the use of only two transmit antennas. It should be noted again that the SNR gains achieved by the proposed coded SOMLD for 6 and $4 \mathrm{~b} / \mathrm{s} / \mathrm{Hz}$ systems of QSM over their uncoded HDMLD schemes, are due, not only to the coding introduced, but also to the effectiveness of the soft-decision over the hard decision techniques.

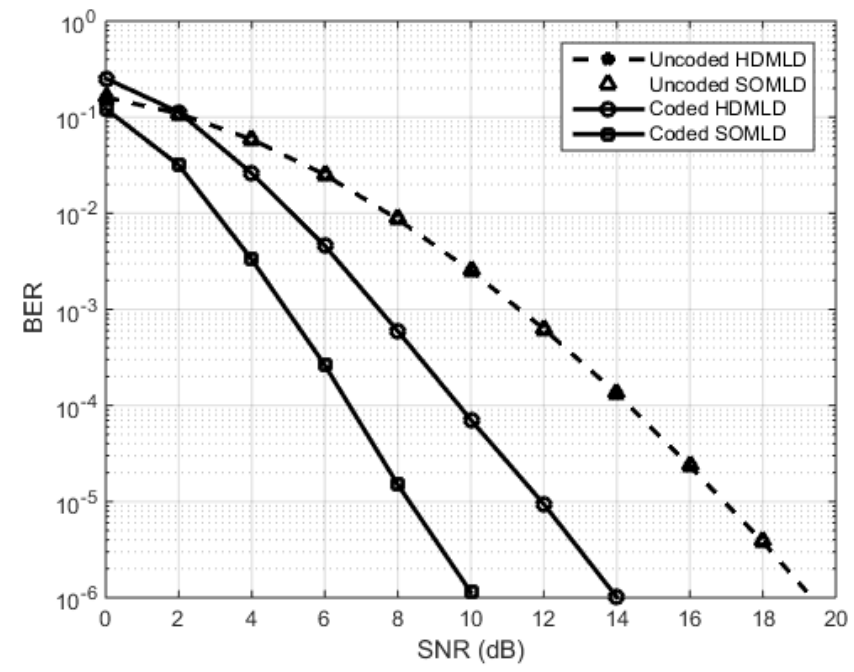

Figure 3: Comparison of error performances for $4 \mathrm{~b} / \mathrm{s} / \mathrm{Hz}$ QSM systems in coded and uncoded channels.

\section{CONCLUSION}

In this paper, a SOMLD for the QSM modulation scheme is proposed. In uncoded channels, the proposed detector matches the optimal error performance of its HDMLD counterpart. In coded channels, the proposed SOMLD yields significant SNR gains over the corresponding conventional coded HDMLD. In comparison to the HDMLD, the proposed SOMLD imposes no additional computational complexity, since a look-up table can be employed to compute the logarithm. Finally, we maintain that a possible future work is 
desired to determine analytical bounds for the proposed SOMLD.

\section{REFERENCES}

Cody, W. and Waite, W. (1980). Software Manual for the Elementary Functions. Prentice Hall, Englewood Cliffs, New Jersey.

Govender, R.; N., Pillay, and H., Xu (2014). Softoutput Space-time Block Coded Spatial Modulation. IET Communications, 8(16): 2786-2796.

Hart, J. F. (1968). Computer Approximations. John Wiley and Sons, New York.

Hu, R.Q. and Qian, Y. (2014). An Energy Efficient and Spectrum Efficient Wireless Heterogeneous Network Framework for 5G Systems. IEEE Communications Magazine, 52(5): 94-101.

Hwang, S.U.; S., Jeon, S., Lee and J., Seo (2009). Softoutput ML detector for Spatial Modulation OFDM Systems. IEICE Electronic Express, 6(19): 1426-1431.

Jeganathan, J., A.; Ghrayeb and L., Szczecinski (2008). Spatial modulation: optimal detection and performance analysis. IEEE Communications Letters, 12(8): 545-547.

Jeganathan, J.; A., Ghrayeb, L. Szczecinski, and A., Ceron (2009). Space shift keying modulation for MIMO channels. IEEE Transactions on Wireless Communications, 8(7): 3692-3703.

Li, C., J.; Wang, Y. Cheng and Y. Huang, (2016). Low-complexity soft-decision aided detectors for coded spatial modulation MIMO systems" EURASIP Journal on Wireless Communications and Networking, 34(1): 1-11.

Li, Q., R.Q.; Hu, Y., Qian, and G., Wu (2012). Cooperative communications for wireless networks: Techniques and Applications in LTE-Advanced Systems. IEEE Wireless Communications, 19(2): 22-29.

Liang, H-W.; R.Y., Chang, W-H., Chung, H. Zhang, and S-Y. Kuo (2012). Bi-Space Shift Keying Modulation for MIMO Systems. IEEE Communications Letters, 16(8): 11611164.

Moon, T.K. (2005). Error Correction Coding: Mathematical Methods and Algorithms. Wiley.

Mesleh, R. Y., H.; Haas, C.W. Ahn, and S. Yun, (2006). Spatial Modulation - A New Low Complexity Spectral Efficiency Enhancing Technique. Paper presented at the First International Conference on Communications and Networking, China, 1-5.
Mesleh, R.Y.; H., Haas, S., Sinanovic, C.W., Ahn, and S., Yun (2008). Spatial Modulation. IEEE Transactions on Vehicular Technology, 57(4): 2228-2241.

Mesleh, R.Y.; Ikki, S.S. and Agoune, H.M. (2014). Quadrature Spatial Modulation - Performance Analysis and Impact of Imperfect Channel Knowledge. Transactions on Emerging Telecommunications Technologies, 28(1): 1-9.

Mesleh, R.Y.; Ikki, S.S., and Agoune, H.M. (2015). Quadrature Spatial Modulation. IEEE Transactions on Vehicular Technology, 64(6): 2738-2742.

Naidoo, N.R.; H.J., Xu, and T.A-M. Quazi (2011). Spatial modulation: optimal detector asymptotic performance and multiple-stage detection. IET Communications, 5(10): 1368-1376.

Naidu, S.; N. Pillay, and H. Xu, (2015). A Study of Quadrature Spatial Modulation. Paper presented at the South African Telecommunications and Network Applications Conference, Western Cape, South Africa, 3-8.

Sklar, B. (2001). Digital Communications: Fundamentals and Applications. Prentice Hall.

Tang, P.T.P. (1991). Table-Lookup Algorithms for Elementary Functions and their Error Analysis. Paper presented at the 10th IEEE International Symposium on Computer Arithmetic, Grenoble, France, 232-236.

Tang, Q., Y.; Xiao, P., Yang, Q., Yu, and S., Li (2013). A New Low-Complexity Near-ML Detection Algorithm for Spatial Modulation. IEEE Wireless Communications Letters, 2(1): 90-93.

Telatar, E. (1999). Capacity of Multi-Antenna Gaussian Channels. European Transactions on Telecommunication, 10(6): 558-595.

Xiao, L.; P., Yang, Y., Xiao, J., Liu, S., Fan B.,, Dong, and S., Li (2016). An Improved Soft-Input Soft-Output Detector for Generalized Spatial Modulation. IEEE Signal Processing Letters, 23(1): 30-34.

Zhao, Y.; P., Yang, Y., Xiao, L. Xiao, B. Dong, and W. Xiang (2017). An Improved Frequency Domain Turbo Equalizer for Single-Carrier Spatial Modulation Systems. IEEE Transactions on Vehicular Technology, 66(8): 75687572.

Zheng, B.; X., Wang, M. Wen, and F. Chen, (2017). Soft Demodulation Algorithms for Generalized Spatial Modulation Using Deterministic Sequential Monte Carlo. IEEE Transactions on Wireless Communications, 16(6): 39533967. 